\title{
PATTERN INTEGRATION
}

R. E. CARR AND J. D. HILL

1. Introduction. Let $f(x)$ be bounded, $a \leqq x \leqq b$, and let the points of discontinuity of $f(x)$ form a set $E$ of zero measure. If the interval $(a, b)$ is subdivided by means of the points $x_{0}, x_{1}, \cdots, x_{n}$, so that $a=x_{0}<x_{1}<\cdots<x_{n}=b$, and if $\xi_{k}$ is chosen from the subinterval $\left(x_{k-1}, x_{k}\right), k=1,2, \cdots, n$, then the existence of

$$
\lim _{n \rightarrow \infty} \sum_{k=1}^{n} f\left(\xi_{k}\right)\left(x_{k}-x_{k-1}\right)
$$

is assured by requiring that the length of the greatest subinterval tend to zero.

If we restrict the summation in (1.1) to a prescribed subset $P$ of the set $N=\{k\}_{1}^{n}$, the resulting limit, providing it exists, will be called the pattern integral of $f(x)$. In general, the existence of this limit and its value depend upon both the manner of subdivision and $P$.

We shall confine our remarks to the case where the manner of subdivision $\Delta$ is given by

$$
\Delta_{1}: x_{k}=a+k \frac{b-a}{n} \quad(k=0,1,2, \cdots, n) .
$$

In this case, with a prescribed subset $P$ of $N$ ( $P$ will also be referred to as the pattern), we form the sum

$$
\text { (P) } \sum_{k=1}^{n} f\left(\xi_{k}\right)\left(x_{k}-x_{k-1}\right),
$$

where the summation is restricted to the subset $P$ of $N$. The limit of (1.2) as $n$ becomes infinite, providing it exists, will be referred to as the special pattern integral

$$
F\left(\Delta_{1}, P\right) \equiv\left(\Delta_{1}, P\right) \int_{a}^{b} f(x) d x \equiv(P) \int_{a}^{b} f(x) d x .
$$

Throughout this paper $(R) \int_{a}^{b} f(x) d x$ will be used to denote a proper Riemann integral, and the statement that $f(x)$ is Riemann integrable in the interval $(a, b)$ will signify that the integral is proper.

2. The principal theorem. We call a pattern fixed if it can be characterized uniquely by a dyadic number

Received by the editors April 6, 1950. 


$$
t=0 . \alpha_{1} \alpha_{2} \alpha_{3} \cdots \alpha_{n} \cdots(2),
$$

where $\alpha_{k}=1$ if the $k$ th term of the sum is to be taken and $\alpha_{k}=0$ if the $k$ th term of the sum is to be omitted. If we cut off the number (2.1) after the first $n$ places we shall refer to the resulting number as

$$
t^{(n)}=0 . \alpha_{1} \alpha_{2} \alpha_{3} \cdots \alpha_{n}(2) .
$$

Without loss of generality we assume the interval of definition of $f(x)$ to be $(0,1)$.

Principal Theorem. Let $f(x)$ be Riemann integrable $0 \leqq x \leqq 1$. Let $P$ be characterized by a given $t$ such that

$$
\lim _{n \rightarrow \infty} \frac{1}{n} \sum_{k=1}^{n} \alpha_{k}=\alpha \text {. }
$$

Then,

$$
\text { (P) } \int_{0}^{1} f(x) d x \equiv \lim _{n \rightarrow \infty} \frac{1}{n} \sum_{k=1}^{n} \alpha_{k} f\left(\xi_{k}^{(n)}\right)=\alpha(R) \int_{0}^{1} f(x) d x .
$$

Proof. (1) Suppose $f(x)=C, 0 \leqq x \leqq 1$. Then $\lim _{n \rightarrow \infty}(1 / n) \sum_{k=1}^{n} \alpha_{k} C$ $=C \lim _{n \rightarrow \infty}(1 / n) \sum_{t=1}^{n} \alpha_{k}=\alpha C$.

(2) Let $0<a<1$, and let $f(x)$ be the step function $f(x)=C_{1}, 0 \leqq x \leqq a$, $f(x)=C_{2}, a<x \leqq 1$. Let the subinterval in which the jump occurs be the $\lambda(n)$ th. Then $\lim _{n \rightarrow \infty}(1 / n) \sum_{k=1}^{n} \alpha_{k} f\left(\xi_{k}^{(n)}\right)=\lim _{n \rightarrow \infty}(1 / n)\left[C_{1} \sum_{k=1}^{\lambda(n)-1} \alpha_{k}\right.$ $+\left(C_{1}\right.$ or $\left.C_{2}\right) \alpha_{\lambda(n)}+C_{2} \sum_{k=1}^{n} \alpha_{k}-C_{2} \sum_{k=1}^{\lambda(n)} \alpha_{k}$.) (Clearly $a-1 / n$ $\leqq[\lambda(n)-1] / n \leqq a \leqq \lambda(n) / n \leqq a+1 / n$, and $\lambda(n) / n \rightarrow a$ as $n \rightarrow \infty$.) Hence

$$
\text { (P) } \int_{0}^{1} f(x) d x=C_{1} \alpha a+C_{2} \alpha-C_{2} \alpha a=\alpha(R) \int_{0}^{1} f(x) d x .
$$

Extension of this result to a step function with $i$ steps follows in the same manner.

(3) Let $f(x)$ be continuous, $0 \leqq x \leqq 1$. There exists a denumerable sequence of step functions $\left\{S_{i}(x)\right\}$ such that, given $\epsilon>0$, one can find an $N_{1}(\epsilon)$ so that $0 \leqq f(x)-S_{i}(x)<\epsilon$ for all $i>N_{1}(\epsilon), 0 \leqq x \leqq 1$. Let $T_{i}(x)=f(x)-S_{i}(x)$. Then $(1 / n) \sum_{k=1}^{n} \alpha_{k} T_{i}\left(\xi_{k}^{(n)}\right) \leqq\left(1\right.$. u.b. of $\left.T_{i}\right)<\epsilon$ for all $i>N_{1}$. Since $f(x)=T_{i}(x)+S_{i}(x), \quad(1 / n) \sum_{k=1}^{n} \alpha_{k} f\left(\xi_{k}^{(n)}\right)=(1 / n)$ . $\sum_{k=1}^{n} \alpha_{k} T_{i}\left(\xi_{k}^{(n)}\right)+(1 / n) \sum_{k=1}^{n} \alpha_{k} S_{i}\left(\xi_{k}^{(n)}\right)$; hence, $0 \leqq(1 / n) \sum_{k=1}^{n} \alpha_{k} f\left(\xi_{k}^{(n)}\right)$ $-(1 / n) \sum_{k=1}^{n} \alpha_{k} S_{i}\left(\xi_{k}^{(n)}\right)<\epsilon$, and for sufficiently large $n$

$$
\left|\frac{1}{n} \sum_{k=1}^{n} \alpha_{k} f\left(\xi_{k}^{(n)}\right)-\alpha(R) \int_{0}^{1} S_{i}(x) d x\right|<2 \epsilon .
$$

Now let $\epsilon \rightarrow 0$, and hence $i \rightarrow \infty$ and $n \rightarrow \infty$. Then 


$$
\text { (P) } \int_{0}^{1} f(x) d x=\alpha(R) \int_{0}^{1} f(x) d x .
$$

(4) Let $f(x)$ be Riemann in tegrable $0 \leqq x \leqq 1 ; f(x)$ is then in tegrable in the sense of Lebesgue. According to Titchmarsh [2], ${ }^{1}$ if $f(x)$ is integrable in the sense of Lebesgue over a finite interval $(a, b)$, we can construct an absolutely continuous function $T(x)$ so that (L) $\int_{a}^{b}|f(x)-T(x)| d x<\eta$, where $\eta$ is arbitrarily small. In our case, $f(x)$ $-T(x)$ and $|f(x)-T(x)|$ are Riemann integrable, so $(R) \int_{0}^{1}[f(x)$ $-T(x)] d x=(L) \int_{0}^{1}[f(x)-T(x)] d x, \quad(R) \int_{0}^{1}|f(x)-T(x)| d x=(L) \int_{0}^{1} \mid f(x)$ $-T(x) \mid d x$. Hence,

$$
\begin{array}{ll}
\left|(R) \int_{0}^{1}[f(x)-T(x)] d x\right|<\eta, & \eta \text { arbitrarily small, } \\
(R) \int_{0}^{1}|f(x)-T(x)| d x<\eta, & \eta \text { arbitrarily small. }
\end{array}
$$

For all $n,\left|(1 / n) \sum_{k=1}^{n} \alpha_{k}\left[f\left(\xi_{k}^{(n)}\right)-T\left(\xi_{k}^{(n)}\right)\right]\right| \leqq(1 / n) \sum_{k=1}^{n} \mid f\left(\xi_{k}^{(n)}\right)$ $-T\left(\xi_{k}^{(n)}\right) \mid$, so from (2.3) we see that by taking $n$ sufficiently large, the quantity $(1 / n) \sum_{k=1}^{n} \alpha_{k}\left[f\left(\xi_{k}^{(n)}\right)-T\left(\xi_{k}^{(n)}\right)\right]$ can be made arbitrarily small. Now, for all $n,(1 / n) \sum_{k=1}^{n} \alpha_{k} f\left(\xi_{k}^{(n)}\right)-(1 / n) \sum_{k=1}^{n} \alpha_{k} T\left(\xi_{k}^{(n)}\right)$ $=(1 / n) \sum_{k=1}^{n} \quad \alpha_{k}\left[f\left(\xi_{k}^{(n)}\right)-T\left(\xi_{k}^{(n)}\right)\right]$, so for $n$ sufficiently large, $(1 / n) \sum_{k=1}^{n} \alpha_{k} f\left(\xi_{k}^{(n)}\right)$ differs from $(1 / n) \sum_{k=1}^{n} \alpha_{k} T\left(\xi_{k}^{(n)}\right)$ by an arbitrarily small quantity. But $\lim _{n \rightarrow \infty}(1 / n) \sum_{k=1}^{n} \alpha_{k} T\left(\xi_{k}^{(n)}\right)=\alpha(R) \int_{0}^{1} T(x) d x$; and by (2.2), (R) $\int_{0}^{1} T(x) d x$ differs from $(R) \int_{0}^{1} f(x) d x$ by an arbitrarily small quantity. Hence, $\lim _{n \rightarrow \infty}(1 / n) \sum_{k=1}^{n} \alpha_{k} f\left(\xi_{k}^{(n)}\right)$ exists and differs from $\alpha(R) \int_{0}^{1} f(x) d x$ by an arbitrarily small quantity, or $\lim _{n \rightarrow \infty}$ $(1 / n) \sum_{k=1}^{n} \alpha_{k} f\left(\xi_{k}^{(n)}\right)=\alpha(R) \int_{0}^{1} f(x) d x$. This completes the proof.

3. Miscellaneous results. The idea of a prescribed variable pattern is suggested by the elementary congruence pattern, $\mathrm{P}: i \equiv n \bmod$ 2. Corresponding to the characterization (2.1) for a fixed pattern, we assume for the variable pattern a sequence of dyadic numbers

$$
\begin{aligned}
& t^{(1)}=0 . \alpha_{1}^{(1)} \quad(2), \\
& t^{(2)}=0 . \alpha_{1}^{(2)} \alpha_{2}^{(2)}(2), \\
& \cdot \cdot \cdot \cdot \cdot \cdot \cdot \cdot, \\
& t^{(n)}=0 . \alpha_{1}^{(n)} \alpha_{2}^{(n)} \cdots \alpha_{n}^{(n)} \quad(2),
\end{aligned}
$$

${ }^{1}$ Numbers in brackets refer to the bibliography at the end of the paper. 
Theorem 3.1. Let $f(x)$ be Riemann integrable, $0 \leqq x \leqq 1$. Let $P$ be characterized by a given sequence $\left\{t^{(i)}\right\}$ such that

$$
\lim _{n_{1}, n_{2} \rightarrow \infty} \frac{1}{n_{1}} \sum_{k=1}^{n_{1}} \alpha_{k}^{\left(n_{2}\right)}=\alpha,
$$

where $n_{1} \leqq n_{2}$ and $0<\lim _{n_{1}, n_{2} \rightarrow \infty}\left(n_{1} / n_{2}\right)$. Then,

$$
\text { (P) } \int_{0}^{1} f(x) d x \equiv \lim _{n \rightarrow \infty} \frac{1}{n} \sum_{k=1}^{n} \alpha_{k}^{(n)} f\left(\xi_{k}^{(n)}\right)=\alpha(R) \int_{0}^{1} f(x) d x .
$$

The proof is almost identical with that of the Principal Theorem. (Actually, the Principal Theorem may be thought of as a special case of Theorem 3.1.)

The following results are either obvious or follow immediately from the Principal Theorem and Theorem 3.1.

TheOREM 3.2. Let $f(x)$ be defined, single-valued, and bounded, $a \leqq x$ $\leqq b$. Let $\alpha=0$. Then $(P) \int_{a}^{b} f(x) d x=0$.

We define $C P$ as the complement of $P$ relative to $N$.

THEOREM 3.3. Let $f(x)$ be Riemann integrable, $a \leqq x \leqq b$. Let (P) $\int_{a}^{b} f(x) d x$ exist. Then

$$
(C P) \int_{a}^{b} f(x) d x=(R) \int_{a}^{b} f(x) d x-(P) \int_{a}^{b} f(x) d x .
$$

Corollary 3.1. Let $f(x)$ be Riemann integrable, $a \leqq x \leqq b$. Let $P$ be the congruence pattern $i \equiv l \bmod p(l, p$ fixed integers). Then $(P) \int_{a}^{b} f(x) d x=(1 / p)(R) \int_{a}^{b} f(x) d x$.

Corollary 3.2. Let $f(x)$ be Riemann integrable, $a \leqq x \leqq b$. Let $P$ be the congruence pattern $i \equiv l_{n} \bmod p\left(p\right.$ fixed integer, $l_{n}$ integer dependent on $n)$. Then $(P) \int_{a}^{b} f(x) d x=(1 / p)(R) \int_{a}^{b} f(x) d x$.

A classical result of Borel [1] may be interpreted as saying that almost all sequences of 0 's and 1's are summable Cesàro of order one to the value $1 / 2$. From this we conclude that, for fixed patterns, almost all special pattern integrals of Riemann integrable functions are equal to $1 / 2$ the corresponding Riemann integrals.

\section{BiBLIOGRAPHY}

1. E. Borel, Les probabilitês dénombrables et leurs applications arithmétiques, Rend. Circ. Mat. Palermo vol. 27 (1909) pp. 247-271.

2. E. C. Titchmarsh, The theory of functions, Oxford, 1932, p. 376.

Michigan State College 